\title{
Lactase non-persistence as a determinant of milk avoidance and calcium intake in children and adolescents
}

\author{
Ricardo $\mathrm{Almon}^{1} *$, Michael Sjöström ${ }^{2}$ and Torbjörn K. Nilsson ${ }^{3,4}$ \\ ${ }^{1}$ Specialist in Family Medicine, Diabetes and Cardiovascular Disease, Genetic Epidemiology, Department of Clinical Sciences, Lund University, \\ 20502 Malmö, Sweden \\ ${ }^{2}$ Unit for Preventive Nutrition, Department of Biosciences and Nutrition, Karolinska Institute, Huddinge, Sweden \\ ${ }^{3}$ Department of Laboratory Medicine, Örebro University Hospital, Örebro, Sweden \\ ${ }^{4}$ Department of Biomedicine, School of Health and Medical Sciences, Örebro University, Örebro, Sweden
}

(Received 26 August 2012 - Final revision received 22 February 2013 - Accepted 5 March 2013)

Journal of Nutritional Science (2013), vol. 2, e26, page 1 of 5

doi:10.1017/jns.2013.11

Abstract

This study examines if lactase non-persistent (LNP) children and adolescents differ from those who are lactase persistent (LP) as regards milk avoidance and $\mathrm{Ca}$ intake. We also studied potential differences in anthropometric features related to obesity, and examined if milk avoidance is associated with lactasepersistence status. Additionally, we aimed to determine if heterozygous subjects showed an intermediary phenotype as regards Ca intake. Furthermore, we tested if LP and LNP influence vitamin D intake. The European Youth Heart Study is an ongoing international, multi-centre cohort study primarily designed to address CVD risk factors. Children ( $n$ 298, mean age 9.6 years) and adolescents ( $n$ 386, mean age 15.6 years) belonging to the Swedish part of the European Youth Heart Study were genotyped for the LCT-13910 C > T polymorphism. Mendelian randomisation was used. Milk avoidance was significantly more common in LNP adolescents (OR 3.2; 95\% CI 1.5, 7·3). LP subjects had higher milk consumption $(P<0 \cdot 001)$. Accordingly, energy consumption derived from milk and $\mathrm{Ca}$ intake was lower in LNP $(P<0.05$ and $P<0 \cdot 001$, respectively). Heterozygous subjects did not show an intermediary phenotype concerning milk consumption. LP or LNP status did not affect vitamin D intake or anthropometric variables. LNP in children and adolescents is associated with reduced intake of milk and some milk-product-related nutritional components, in particular Ca. This reduced intake did not affect the studied anthropometric variables, indicators of body fat or estimated vitamin D intake. However, independently of genotype, age and sex, daily vitamin D intake was below the recommended intakes. Milk avoidance among adolescents but not children was associated with LNP.

Key words: Dairy products: Calcium: Vitamin D: LCT-19310 C > T polymorphism: Mendelian randomisation: Milk avoidance: Nutrigenetics

Lactase non-persistence (LNP) is as an autosomal recessive trait leading to down-regulation of lactase activity in the intestinal mucosa and to maldigestion of lactose ${ }^{(1)}$. Milk and some dairy products contain lactose, a disaccharide hydrolysed by the enzyme lactase-phlorizin hydrolase to glucose and galactose in the brush border of the small intestine. LNP is widespread throughout the world and plays an important role in the everyday work of general practitioners, gastroenterologists and paediatricians ${ }^{(2)}$.
The diagnosis of lactase persistence (LP) and LNP has by definition been based on the measurement of lactase, sucrase and maltase activities and the lactase to sucrase ratio in intestinal biopsies ${ }^{(3)}$. This is an invasive technique that is not suitable for primary exploration of abdominal complaints or for large-scale population studies of the effect of LNP on anthropometric or nutritional variables.

Enattah et al. identified in 2002 the position of the LP-associated allele as a single nucleotide polymorphism C

Abbreviations: LNP, lactase non-persistence; LP, lactase persistence.

* Corresponding author: Dr. R. Almon, fax + 4619 6025797, email ricardo.almon@med.lu.se

(C) The Author(s) 2013. The online version of this article is published within an Open Access environment subject to the conditions of the Creative Commons Attribution-NonCommercial-ShareAlike licence $<\mathrm{http}: / /$ creativecommons.org/licenses/by-nc-sa/2.5/>. The written permission of 
$>$ T located $13.9 \mathrm{~kb}$ upstream of the first ATG of LCT. The single nucleotide polymorphism is located in intron 13 of the MCM6 gene. Homozygosity for the C allele (LCT-13910 CC) shows for all practical purposes a complete association with LP in populations of European descent ${ }^{(4,5)}$. Molecular epidemiological studies have shown that the prevalence of LNP assessed by genotyping is consistent with previously published phenotypically determined epidemiological data in more than seventy countries ${ }^{(6)}$. Different test methods in the diagnosis of LNP, including the standard physiological tests, are useful at different levels of health care organisation or symptomatology ${ }^{(7)}$.

The majority of heterozygous individuals, having intermediary levels of lactase activity in intestinal biopsies, are traditionally thought to produce sufficient lactase to be classified by the standard physiological tests as $\operatorname{LP}^{(8)}$, but pre-2002 studies can hardly be regarded as conclusive. One of the aims of this study was to look for a potential gene-dose effect of the LCT-13910 $\mathrm{C}>\mathrm{T}$ polymorphism with respect to some nutritional variables in children and adolescents.

Milk and other dairy products are major dietary sources of $\mathrm{Ca}$ in Western $\operatorname{diets}^{\left({ }^{(9)}\right.}$ and the diet of LNP individuals may be restricted as regards milk intake. The main hypothesis tested in this study was that LNP might influence the intake of $\mathrm{Ca}$ and vitamin $\mathrm{D}$ through its effects on milk consumption, since there is mandatory fortification of milk with vitamin D in Sweden. We were also interested in seeing whether milk avoidance, which is partly a lifestyle and behavioural/attitudinal variable, was associated with the LCT-13910 C > T polymorphism.

\section{Methods}

\section{Population}

Blood samples were obtained from 684 children (334 girls and 350 boys) belonging to the Swedish part of the European Youth Heart Study, which is a cross-sectional school-based study of risk factors for future CVD among children 9-10 years old and adolescents 15-16 years old. Mean ages in the Swedish sample were 9.6 years and 15.6 years, respectively. Sampling procedures and participation rates have been described previously ${ }^{(10)}$.

Height, weight, hip and waist circumference were directly measured by standardised procedures. BMI was calculated as weight/height ${ }^{2}\left(\mathrm{~kg} / \mathrm{m}^{2}\right)$. The consumption of milk was assessed by an interviewer-mediated 24 -h recall. A qualitative food record completed on the day before the interview served as checklist for the data obtained by $24-\mathrm{h}$ recall. A food atlas was used to estimate portion sizes. Dietary data were processed by StorMats (version 4.02; Rudans Lättdata) and analysed using the Swedish National Food database (version 99.1). Total $\mathrm{Ca}$ intake was calculated in $\mathrm{mg} / \mathrm{d}$ and vitamin $\mathrm{D}$ intake was calculated in $\mu \mathrm{g} / \mathrm{d}$.

For the genetic analysis genomic DNA was isolated from the EDTA whole blood samples from the individuals with the QIAamp DNA Blood Mini Kit spin procedure. The DNA fragment spanning the $-13910-\mathrm{C} / \mathrm{T}$ polymorphic site was genotyped by pyrosequencing, using a PSQ96 SNP reagent Kit and a PSQ 96MA system (Pyrosequencing AB) PSQ96MA 2.0.1 software. The procedure has been previously described in detail $^{(8)}$.

\section{Statistical analysis}

Statistical analyses were performed with the Statistical Package for Social Sciences (SPSS, version 13.0 for Windows; SPSS Inc., Chicago). The data are presented as means and standard deviations in Table 1. The Student's $t$ test for differences was used to compare the LCT-13910 TT and LCT-13910 CT and CC genotypes concerning nutritional and anthropometric data. Data were checked for normality. Quantitative effects of the LCT-13910 C > T genotype on the selected anthropometric and food intake related variables were tested in three-way ANOVA models with the fixed factors age group (children/ adolescents), sex (girls/boys) and LCT-13910 C > T genotype with the levels TT/CT $v$. CC. A qualitative variable 'complete milk avoidance' (yes/no) was generated from the continuous variable, intake of milk $(\mathrm{g} / \mathrm{d})$, present in the dietary survey. The OR for complete milk abstinence was tested separately in children and adolescents by logistic regression with sex and LCT-13910 C > T genotype with the levels TT/CT $v$. $\mathrm{CC}$ as covariates.

\section{Mendelian randomisation}

Since the carriage of the LCT-13910 C $>\mathrm{T}$ polymorphism is subject to random assortment of maternal and paternal alleles at the time of gamete formation, associations between LCT genotypes and our observational data should not be subject to reverse causality. This is a basic assumption of Mendelian randomisation $^{(11,12)}$, which examines causal effects of modifiable exposures on disease in genetic epidemiology. A functional genetic variant, in our study LCT-13910 C > T polymorphism, acts as a proxy for modifiable lifetime exposure patterns (milk consumption). The LCT-13910 C > T polymorphism is known to influence milk consumption ${ }^{(13)}$. According to Mendel's second law of independent assortment, the inheritance of one trait is independent of the inheritance of other traits. Thus, associations between genetic variants and outcome are not generally confounded by behavioural, physiological or environmental exposures, and observational studies of genetic variants have similar properties to intention-to-treat analyses in randomised controlled trials ${ }^{(11,12,14,15)}$.

This study was conducted according to the guidelines laid down in the Declaration of Helsinki and all procedures were approved by the Research Ethics Committees of Örebro County Council and Huddinge University Hospital. Parents and 15-year-olds gave specific written informed consent to participate in the present study.

\section{Results}

Distribution of the LCT-13910 C > T genotype showed that the CT and T'T genotypes, which are associated with LP, were found in 273 and 317 subjects, respectively. The 


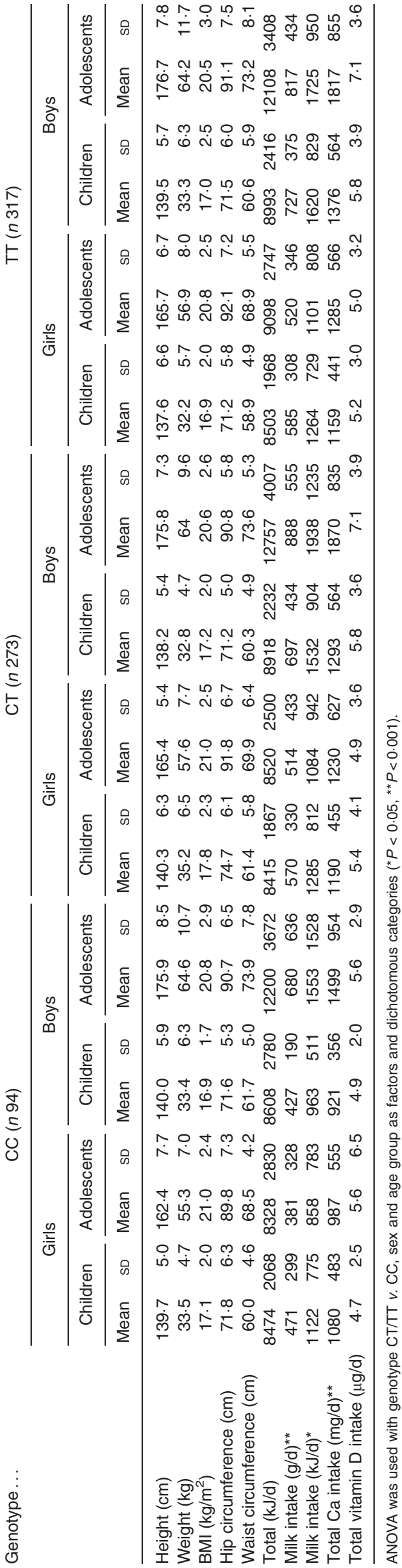

Table 2. Frequency distribution for complete milk avoidance by LCT-13910 C > T genotype and sex among the studied children and adolescents

\begin{tabular}{|c|c|c|c|c|}
\hline \multirow[b]{3}{*}{ Genotype } & \multicolumn{4}{|c|}{ Milk abstinence ( $n$ 39) } \\
\hline & \multicolumn{2}{|c|}{ Children ( $n$ 5) } & \multicolumn{2}{|c|}{ Adolescents ( $n$ 34) } \\
\hline & Girls & Boys & Girls & Boys \\
\hline $\mathrm{CC}$ & $0 / 19$ & $0 / 18$ & $6 / 28$ & $5 / 29$ \\
\hline $\mathrm{CT} / \mathrm{TT}$ & $2 / 119$ & $3 / 142$ & $15 / 168$ & $8 / 161$ \\
\hline
\end{tabular}

genotype LCT-13910 CC, associated with LNP, was found in ninety-four subjects. The baseline characteristics including selected anthropometric and milk intake-related variables of these three genotypes are shown in Table 1. There were no statistically significant differences between the genotypes CT and TT in any of the selected variables. Intermediary phenotypes for LCT-13910 C > T heterozygous subjects were thus not observable with respect to these anthropometric and food intake data in the studied population. In subsequent analyses the effect of the LCT-13910 C > T polymorphism was tested by using two levels: TT/CT $v$. CC.

As shown in Table 1, the LCT-13910 CC genotype was associated with statistically significantly lower levels of the following variables: milk intake, energy intake from milk and $\mathrm{Ca}$ intake. No statistically significant interactions of the LCT-13910 C > T genotype with age and sex for the selected variables were found. Height, weight, BMI, hip circumference and waist circumference as well as total daily energy intake and vitamin $\mathrm{D}$ intake did not differ significantly between the LCT-13910 C > T genotypes in these ANOVA models.

The odds for milk avoidance due to the subjects' LCT-13910 C > T genotype was tested (Table 2). Only five children avoided milk, and none of these had the CC genotype. Among adolescents, milk avoidance was more frequent (n 34): the OR for subjects with LNP compared with LP subjects was $3.2(95 \%$ CI $1.5,7.3, P=0.003)$, with sex and LCT-13910 C > T genotype as covariates in the model. Milk protein allergies were not reported in the studied population.

\section{Discussion}

The main finding was that LNP subjects had a lower milk consumption, a lower daily energy intake based on these products and a lower $\mathrm{Ca}$ intake. These differences did not translate into any difference in total daily energy intake. Furthermore, no signs of increased indicators of body fat in LP individuals could be observed.

No evidence was found for a gene-dose effect of the LCT-13910 C > T mutation in the studied sample. An intermediary phenotype could not be identified with respect to the studied nutritional and anthropometric variables (Table 1), in accordance with the traditional view of 'lactose intolerance' as a recessive trait. However, we cannot rule out such a gene-dose effect in adults, in subjects of other populations, or with respect to other possible LCT-dependent 
phenotypes not yet studied. Hence, further studies are needed to verify the absence or presence of a gene-dose effect.

The other main finding was that the LCT-13910 C > T polymorphism influences complete milk avoidance among adolescents (Table 2). The lower milk intake of LNP adolescents could be a cause of concern as trends of replacement of milk by soft drinks have been reported, and appear to be detrimental to bone health ${ }^{(16,17)}$. Milk constitutes a basic source of dietary $\mathrm{Ca}$ in most Western diets, and adequate $\mathrm{Ca}$ intakes are directly related to the consumption of these food items. Frequently, milk is being replaced as a beverage of choice by sweetened and carbonated soft drinks and juices ${ }^{(18,19)}$. In addition, limiting milk in the diet might in some cases lead to the necessity for dietary adjustments beyond meeting only $\mathrm{Ca}$ requirements. The critical role of $\mathrm{Ca}$ in human health has been recognised for many years, as reflected by a long history of $\mathrm{Ca}$ recommendations ${ }^{(20)}$. LP children and adolescents consumed significantly more milk than LNP subjects, and LP did not reveal any tendency towards increased BMI or other indicators of obesity. This observation has been confirmed by an earlier study performed by the authors on the same sample using body fat percentage as a variable $^{(21)}$.

Vitamin D intake was in both LNP and LP subjects below the recommended intakes issued by the Swedish National Food Agency (updated 9 October 2012). The Swedish National Food Agency's recommended intake for vitamin D is $7.5 \mu \mathrm{g}$ per $\mathrm{d}$ for the studied sample of children and adolescents. Independently of genotype, age group or sex all subjects did not meet recommended intakes for vitamin $\mathrm{D}$ (Table 1).

Limitations of this study are sample size and age of onset of LP that can show a wide regional and ethnic variation. Genetically programmed down-regulation of lactase-phlorizin hydrolase synthesis has been observed starting from the second year of life. The majority of Thai children manifest LNP by the age of 2 years and in black populations genetically determined lactose intolerance manifests between 1 and 8 years. In white populations, it is rarely seen before 5 years of age ${ }^{(22,23)}$.

In this study two different age groups were compared, 9-year-old children (mean age 9.6 years) and 15 -year-old adolescents (mean age 15.6 years). Thus, it can be assumed that the majority of children and almost all adolescents with the LCT-13910 CC genotype had developed manifest LNP at the time of inclusion in this study. However, the correlation between manifest LNP and self-reported 'lactose intolerance' has been suggested earlier to be poor in a few other studies $^{(24-26)}$.

Almost all children and many adolescents with the CC genotype consumed some amount of milk even though having LNP status. This is compatible with many studies showing that LNP subjects can tolerate a certain amount of lactose intake per $\mathrm{d}^{(27-29)}$. Other individuals do not consume milk and dairy products because of health reasons such as milk protein allergies, perceived 'lactose-intolerance', taste preferences or dietary culture and fashion.

Mendelian randomisation was used in this study. The main assumption in this study is that LP (lactose tolerant) individuals consume on average significantly more milk than LNP (lactose intolerant) individuals throughout their lifetime, and not only at the moment dietary intakes were assessed. If this assumption is correct, the LCT-13910 C > T polymorphism can be used as a proxy measure for lifetime exposure to milk and dairy intake patterns. Cultural influences on milk consumption might be able to override the discomforts consequent on milk ingestion in lactose-intolerant individuals. This, nevertheless, could not be observed in our sample. Neither have we been able to observe this in another sample representative of the general population of the Canary Islands in Spain ${ }^{(30)}$.

In summary, LNP in children and adolescents is associated with reduced intake of milk and particularly $\mathrm{Ca}$. This reduced intake did not affect the studied anthropometric variables with respect to indicators of body fat. Reduced intake of Ca could be compensated by consumption of dairy products with lower amounts of lactose than milk. Heterozygous subjects did not show an intermediary phenotype. Estimated vitamin D intake unexpectedly did not differ between LP and LNP subjects, although milk is regularly fortified with vitamin $\mathrm{D}$ in Sweden. Independently of genotype, age and sex, overall daily vitamin $\mathrm{D}$ intake was below recommended intakes.

\section{Acknowledgements}

This study was supported by grants from Örebro Läns Landstings Forskningskommitté and by Nyckelfonden, Örebro, Sweden. Stockholm County Council, Sweden is acknowledged for the collection of blood samples. We are grateful to Associate Professor Olle Carlsson for statistical advice. There are no conflicts of interest to declare. R. A. wrote the paper. M. S. provided the data from the European Young Heart Study. T. K. N. performed the genetic analyses. M. S., T. K. N. and R. A. analysed the data and designed the study. All authors read and approved the final manuscript.

\section{References}

1. Sahi T, Isoloski M, Jussila J, et al. (1973) Recessive inheritance of adult-type lactose malabsorption. Lancet ii, 823-826.

2. Apley J \& Naish N (1958) Recurrent abdominal pains: a field survey of 1000 school children. Arch Dis Child 33, 165-170.

3. Messer M \& Dahlqvist A (1966) A one-step ultramicro method for the assay of intestinal disaccharidases. Anal Biochem 14, 376-392.

4. Enattah NS, Sahi T, Savilahti E, et al. (2002) Identification of a variant associated with adult-type hypolactasia. Nat Genet 30, 233-237.

5. Rasinperä H, Kuokkanen M, Kolho KL, et al. (2005) Transcriptional downregulation of the lactase (LCT) gene during childhood. Gut 54, 1660-1661.

6. Järvelä IE (2005) Molecular diagnosis of adult-type hypolactasia (lactase non-persistence). Scand J Clin Lab Invest 65, 535-539.

7. Arola H (1994) Diagnosis of hypolactasia and lactose malabsorption. Scand J Gastroenterol Suppl 202, 26-35.

8. Nilsson TK \& Johansson CA (2004) A novel method for diagnosis of adult hypolactasia by genotyping of the $-13910 \mathrm{C} / \mathrm{T}$ polymorphism with pyrosequencing technology. Scand I Gastroenterol 39, 287-290.

9. Rafferty K \& Heaney RP (2008) Nutrient effects on the calcium economy: emphasizing the potassium controversy. J Nutr 138, 166S-171S.

10. Wennlof AH, Yngve A \& Sjöstrom M (2003) Sampling procedure, participation rates and representativeness in the Swedish part of the 
European Youth Heart Study (EYHS). Public Health Nutr 6, 291-299.

11. Davey Smith G \& Ebrahim S (2003) 'Mendelian randomization': can genetic epidemiology contribute to understanding environmental determinants of disease? Int J Epidemiol 32, 1-22.

12. Davey Smith G \& Ebrahim S (2005) What can Mendelian randomisation tell us about modifiable behavioural and environmental exposures? BMJ 330, 1076-1079.

13. Swallow DM (2003) Genetics of lactase persistence and lactose intolerance. Annu Rev Genet 37, 197-219.

14. Ebrahim S \& Davey Smith G (2008) Mendelian randomization: can genetic epidemiology help redress the failures of observational epidemiology? Hum Genet 123, 15-33.

15. Lewis SJ (2010) Mendelian randomization as applied to coronary heart disease, including recent advances incorporating new technology. Circulation: Cardiovasc Genet 3, 109-117.

16. Whiting SJ, Vatanparast H, Baxter-Jones A, et al. (2004) Factors that affect bone mineral accrual in the adolescent growth spurt. J Nutr 134, 696S-700S.

17. Tolonen S, Laaksonen M, Mikkilä V, et al. (2011) Lactase gene c/t (-13910) polymorphism, calcium intake, and pQCT bone traits in Finnish adults. Calcif Tissue Int 88, 153-161.

18. McGartland C, Robson PJ, Murray L, et al. (2003) Carbonated soft drink consumption and bone mineral density in adolescence: the Northern Ireland Young Hearts project. J Bone Miner Res 18, 1563-1569.

19. Storey ML, Forshee RA \& Anderson PA (2004) Associations of adequate intake of calcium with diet, beverage consumption, and demographic characteristics among children and adolescents. J Am Coll Nutr 23, 18-33.

20. Mertz W (2000) Three decades of dietary recommendations. Nutr Rev 58, 324-331.
21. Almon R, Patterson E, Nilsson TK, et al. (2010) Body fat and dairy product intake in lactase persistent and non-persistent children and adolescents. Food Nutr Res 54 (epublication 16 June 2010).

22. Simoons FJ (1980) Age of onset of lactose malabsorption. Pediatrics 66, 646-648.

23. Keusch GT, Troncale FJ, Thavaramara B, et al. (1969) Lactase deficiency in Thailand: effect of prolonged lactose feeding. $A m \mathrm{~J}$ Clin Nutr 22, 638-641.

24. Gugatschka M, Dobning H, Fahrleitner-Pammer A, et al. (2005) Molecularly-defined lactose malabsorption, milk consumption and anthropometric differences in adult males. QJM 98, 857-863.

25. Enattah N, Pekkarinen T, Välimäki MJ, et al. (2005) Genetically defined adult-type hypolactasia and self-reported lactose intolerance as risk factors of osteoporosis in Finnish postmenopausal women. Eur J Clin Nutr 59, 1105-1111.

26. Lehtimäki T, Hemminki J, Rontu R, et al. (2006) The effects of adult-type hypolactasia on body height growth and dietary calcium intake from childhood into young adulthood: a 21-year follow-up study - the Cardiovascular Risk in Young Finns Study. Pediatrics 118, 1553-1559.

27. Savaiano DA, Boushey CA \& McCabe GP (2006) Lactose intolerance symptoms assessed by meta-analysis: a grain of truth that leads to exaggeration. J Nutr 136, 1107-1113.

28. Suarez FL, Savaiano D, Arbisi P, et al. (1997) Tolerance to the daily ingestion of two cups of milk by individuals claiming lactose intolerance. Am J Clin Nutr 65, 1502-1506.

29. Scrimshaw NS \& Murray EB (1988) The acceptability of milk and milk products in populations with a high prevalence of lactose intolerance. Am J Clin Nutr 48, 1079-1159.

30. Almon R, Alvarez-Leon EE, Engfeldt P, et al. (2010) Associations between lactase persistence and the metabolic syndrome in a crosssectional study in the Canary Islands. Eur J Nutr 49, 141-146. 